\title{
Dynamic heating control by infrared thermography of prepreg thermoplastic CFRP designed for reinforced concrete strengthening
}

\author{
by L-D. Théroux*, J. Dumoulin* and J.L. Manceau* \\ * LUNAM Université, IFSTTAR, COSYS/SII, Route de Bouaye, CS4, F-44340, Bouguenais, France \\ jean.dumoulin@ifsttar.fr
}

\begin{abstract}
In civil engineering, reinforced concrete repair by CFRP is a strengthening technique that has proven successfully in the past. The present study is aimed at using thermoplastic CFRP sheets applied and glued under heat. In this research framework, active thermography is used to accomplish two roles: control of the operating temperature of the thermoplastic CFRP sheets during the installation process and evaluation of the bonding quality after welding. The paper presents results obtained in laboratory with a dedicated test bench coupled with numerical simulations of the process.
\end{abstract}

\section{Introduction}

Failing and deteriorating concrete structure has always been a major problem in civil engineering. As many structures are growing older, the stakes are now higher than ever. Retrofitting with CFRP has become a popular solution and has proven its efficiency [1-2]. Generally, applications are carried out by using thermosetting resins to maintain the link between CFRP and concrete. However, they present inconveniences of dangerousness in handling during the application. These resins are also hazardous for the health of the operators exposed to the emanations of these products, especially in confined or unventilated environments. The present study, part of the REPTILES project (lead by FREYSSINET INTERNATIONAL \& Cie), is aimed at solving these problems by the use of pre-impregnated (prepreg) thermoplastic CFRP sheets. It also aims at developing an automatic process that installs this thermoplastic CFRP on a primer covered the concrete surface. To install the CFRP sheets, the prepreg glue must first be melted to adhere to the surface (welding phase).

The goal of this research is to study a dynamic heating solution and then monitor the process with infrared thermography [3-6]. The main constrain is the installation speed of up to $10 \mathrm{~cm} / \mathrm{s}$ in working operative conditions. The CFRP must be heated at a temperature at least equal to the point of melting of the thermoplastic binder. It must be taken into account that the ambient condition may vary and could impact the heating process. Furthermore, movements during the installation process may cause variation in the distance between the CFRP and the optical heating solution studied. In this regard, to make sure the temperature of the CFRP is neither too high nor too low, the heating process will be monitored by infrared thermography.

\section{Problem positioning}

\subsection{Infrared measurements and phase change considerations}

As mentioned in the introduction, the thermoplastic CFRP need to be heated while scrolling. Then, it will be glued to the primer that was applied previously to the concrete surface. To optimize the heating process and to control the quality of the gluing, a non-destructive system will be put into place. Active infrared thermography will have to ensure that the CFRP is heated enough, but also to evaluate the bonding of the composite sheet after it has been glued on the structure. The temperature, measured by infrared camera in our specific context of application, is mainly associated to the direct heat flux [7] radiated by the composite sheet according to the relation:

$$
\phi=\varepsilon \cdot \sigma \cdot A \cdot T^{4}
$$

In this equation, the emissivity $\epsilon$ is a parameter that depends on the material temperature and may also change in function of the wavelength and the observation angle. To optimize the heating process and the thermography monitoring, it is necessary to determine the evolution of this parameter at least versus temperature. A simplified approach will be proposed and presented in paragraph 3.

Another important aspect for the numerical simulation is the effect of latent heat. Differential Scanning Calorimetry (DSC) measurements have shown that the phase transition of the thermoplastic glue cause a notable amount of latent heat to be absorbed. During the solidification, when the glue cools down, latent heat is release. To account the effect of the latent heat behavior with temperature evolution during the phase change, enthalpy variation will be introduced [8] for the glue in the numerical simulations according to eq. 2 . 


$$
[\Delta H]_{T_{p t}-\Delta T_{\min }}^{T_{p t}+\Delta T_{\max }}=\int_{T_{p t}-\Delta T_{\min }}^{T_{p t}+\Delta T_{\max }} C_{p} d T
$$

Where:

$\Delta H$ is the change in enthalpy associated to the latent heat

$C_{p}$ is the thermal capacity at constant pressure

$T_{p t}$ is the temperature of the peak phase transition

$\Delta T_{\min }$ and $\Delta T_{\max }$ are the maximum and minimum temperature limits of the temperature interval associated to the phase change phenomenon

These parameters were defined by analyzing the DSC thermograms carried out for the thermoplastic (see paragraph 3). In a first approach, the shape of the enthalpy change is approximated by a Gaussian.

\subsection{Heating considerations for the welding}

To heat the CFRP to the required temperature, multiples heating methods are at our disposal. The first criterion is that it needs to be a contactless method. This is because if the CFRP must be heated until the glue melt, if there is contact, the glue could get stuck on the surface. Among the contactless methods, four have retained our attention; by induction, laser, plasma torch and heat lamp. Induction and laser were both not selected due to practical issue considered in the design of the final automated process. The use of plasma torch is not recommended since its heating is localized and is not easily applicable to heat a wide area in our final application configuration. Finally, heating by infrared lamps was chosen since it is the safest as well as easily implemented.

To estimate the heat exchange between the heating lamps and the composite sheet we assumed in a first approach that we are at each time step at the local thermal equilibrium and we have considered radiative heat exchange between two grey diffuse surfaces. According to these hypothesis, the net heat flux [7] can be calculated from the following equation:

$$
\phi_{1 \rightarrow 2}=\frac{\sigma\left(T_{1}^{4}-T_{2}^{4}\right)}{\frac{1-\varepsilon_{1}}{\varepsilon_{1} A_{1}}+\frac{1}{F_{1 \rightarrow 2} A_{1}}+\frac{1-\varepsilon_{2}}{\varepsilon_{2} A_{2}}}
$$

The radiative heat exchange between both surfaces depends on the view factor $F$ :

$$
F_{1 \rightarrow 2}=\frac{1}{A_{1}} \int_{A_{1}} \int_{A_{2}} \frac{\cos \theta_{1} \cos \theta_{2} d A_{1} A_{2}}{\pi d^{2}}
$$

The choice of the heating lamps will depend on the absorptivity, reflectivity and transmission of the CFRP sheet in function of the wavelength. For an optimal heating, it is preferable to use a lamp that radiate in the spectrum were the absorptivity of the composite is at its highest. After first spectral characterization by using Fourier Transform Spectrometer from UV to IR [9], our choice of type of lamps tumbled on short-wave lamps. These types of lamps radiate mostly in the band containing the wavelength from 0.2 to $2 \mu \mathrm{m}$.

\section{Characterization}

\subsection{Thermal properties}

To optimize the heating process and to carry out first numerical simulations, thermal properties of materials were estimated in laboratory using the "DICO" experimental set-up developed at CERTES laboratory [10]. The thermal properties of the composite (assumed homogeneous in a first approach) and of the primer are summarized in the

\begin{tabular}{|c|c|c|c|c|c|c|}
\hline & Thickness & Temperature & Density & $\begin{array}{c}\text { Thermal } \\
\text { Conductivity }\end{array}$ & Thermal Diffusivity & $\begin{array}{l}\text { Thermal } \\
\text { Capacity }\end{array}$ \\
\hline Units & {$\left[10^{-3} \mathrm{~m}\right]$} & {$[\quad \square \mathrm{C}]$} & {$\left[\mathrm{kg} \cdot \mathrm{m}^{-3}\right]$} & {$\left[\mathrm{W} \cdot \mathrm{m}^{-1} \cdot \mathrm{K}^{-1}\right]$} & {$\left[10^{-7} \mathrm{~m}^{2} \cdot \mathrm{s}^{-1}\right]$} & {$\left[\mathrm{J} \cdot \mathrm{kg}^{-1} \cdot \mathrm{K}^{-1}\right]$} \\
\hline $\begin{array}{l}\text { Primer } \\
\text { Prepreg CFRP }\end{array}$ & 2.8 & 20 & 1314 & 0.26 & 1.44 & 1371 \\
\hline multilayer & 1.8 & 18.1 & 1420 & 0.7 & 4.8 & 922 \\
\hline
\end{tabular}
following tables.

Table 1. Material properties of the primer and the pre-impregnated CFRP sheet used for the simulations.

The remaining properties to be known are the composite emissivity in function of the temperature and the latent heat caused by the phase change of the thermoplastic glue. 


\subsection{Emissivity}

To evaluate the thermal properties of the composite, various test were conducted. Spectral Emissivity and thermal properties were determined in lab at ambient temperature. But in our study, the emissivity of the CFRP in function of the temperature is a parameter that must be known to efficiently control the temperature during the process. Since the emissivity variation with temperature is unknown, a characterization experiment was put into place. The idea is to have a reference of know emissivity and temperature and compare digital level measured with infrared camera with the one from the CFRP at the same temperature. This method inspired from [11] is also called the flux equalization and was adapted in [12] for paint emissivity evaluation with temperature by using infrared thermography. To do this, the experimental setup consists of an aluminum support on which the top surface was painted in black. The paint used is of known emissivity and have a diffuse reflectivity. Incrusted in the support is a shallow hole in which the CFRP is installed. The hole is covered in a PTFE tissue (covering) that prevents the composite sheet to glue to the aluminum support. Multiples thermocouples coated in conductive thermal grease are inserted in the aluminum support as well as on and bellow the CFRP sheets. The support is installed on a heating plate with an adjustable temperature as presented in Fig. 1.
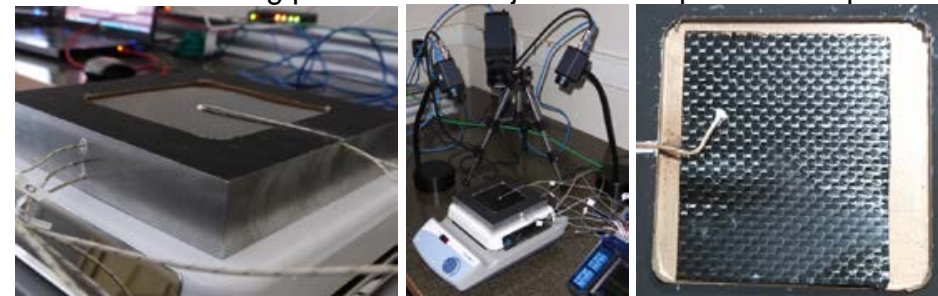

Fig. 1. Photos of the experimental setup for the characterization of emissivity of the composite. On the right, the picture of the partially melted composite sheet after heating.

As we can see in the third picture of Fig. 1 (right) the texture of the CFRP sheet (after heating) has changed by comparison to its aspect before melting (upper part of the photo). For this sample, this was caused by the fact the not all the surface of the sheet was in contact with the support. This caused the lower area to not be melted compared to the rest of the sample. It can be seen that portion of CFRP sheets that have melted glue is not as reflective in the visible spectrum as non-melted prepreg composite.

The melting also caused a deformation in the sample that caused non-uniform heating and can be best examined in the thermal images presented in the following Fig. 2.

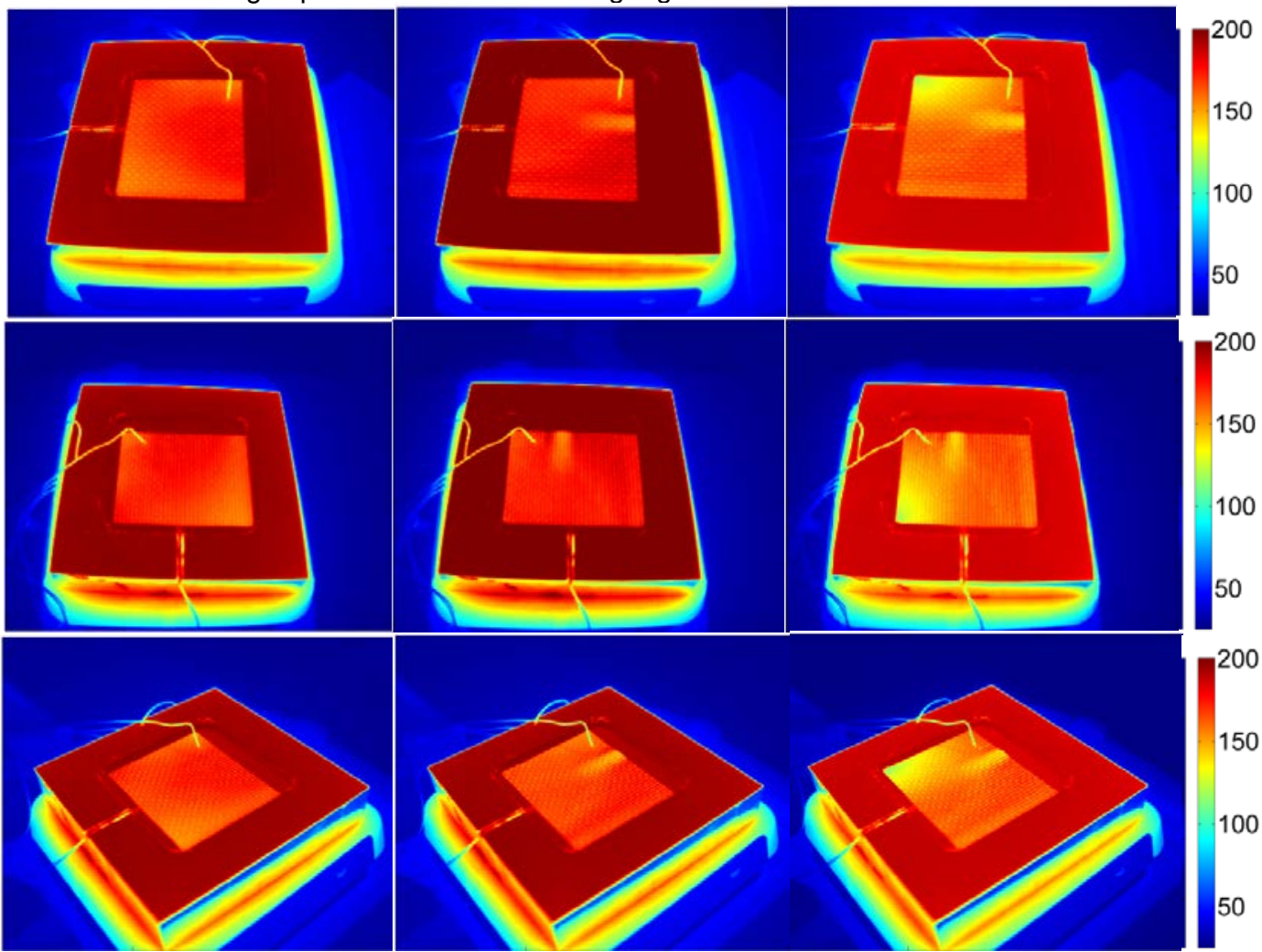

Fig. 2. The thermal images for both A35 infrared camera and for the cooled infrared camera SC655 on the bottom. 
This experiments was also used to make comparative testing between two types of uncooled IRFPA in the LWIR bandwidth (see set up picture in the middle of Fig 1) :

- $\quad$ One infrared camera FLIR SC655 with 640X480 pixels detector matrix with a pixel pitch of $17 \mu \mathrm{m}$

- Two infrared camera FLIR A35 with 320X240 pixels detector matrix with a pixel pitch of $25 \mu \mathrm{m}$

To investigate the different behavior of the A35 cameras and the cooled SC655 camera, we take a look at the difference between temperature profiles (thermograms). The temperature evolution was calculated as the mean over a region of interest containing only the painted surface of the support. The difference in temperature between the profiles extracted from each infrared camera is presented in Fig. 3.
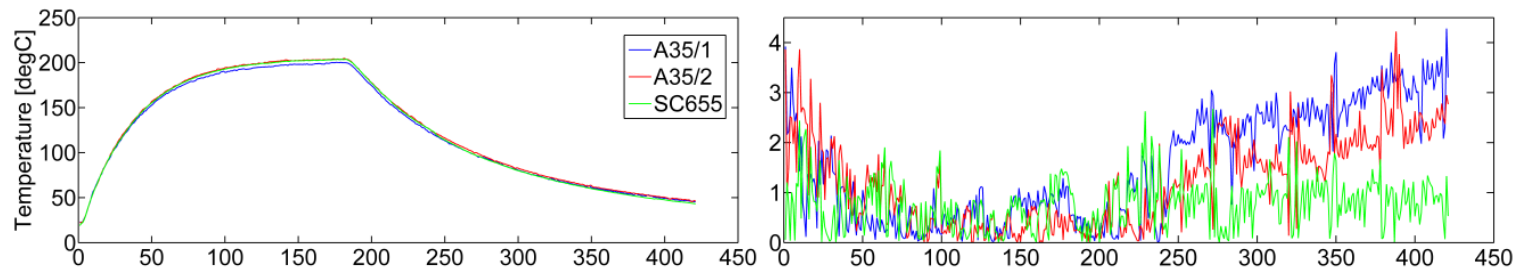

Fig. 3. Thermograms extracted for both A35 infrared camera and for the SC655 infrared camera (left) and differences between A35 and SC655 thermograms (right). The $x$ axis is the frames number with one frame acquisition each 30 sec.

Looking at the right graph of Fig. 3., the temperature difference between each of the infrared camera is very important for the first many and last frames. For these frames, the temperature difference can reach up to $4^{\circ} \mathrm{C}$. However, for the $50^{\text {th }}$ to the $200^{\text {th }}$ frames, the difference is lower than $1^{\circ} \mathrm{C}$. This particular behavior can be explained by examining the right graph of Fig. 3. It can be observed that for high temperatures, the difference between the measurements of the infrared camera is lower. This is coherent with the fact that the infrared cameras were configured to measure high temperatures (large scale calibration range). The effect of the temperature difference on the error of the emissivity can be calculated. For example, in the worst case where the temperature of the CFRP is at the room temperature of $20^{\circ} \mathrm{C}$ and the maximum error on the measurement (for low temperature) is $4^{\circ} \mathrm{C}$ which would results in an estimation error of $20 \%$ on the emissivity. On the other hand, if we only consider high temperatures, the maximum error is $1^{\circ} \mathrm{C}$. For a temperature of $150^{\circ} \mathrm{C}$, this corresponds to an emissivity estimation error of approximately $0.5 \%$.

Taking the temperature measurements from the infrared cameras and by using the temperatures of the thermocouples, it is possible to calculate a first direct estimation of the emissivity of the composite. Results are presented in Fig.4.

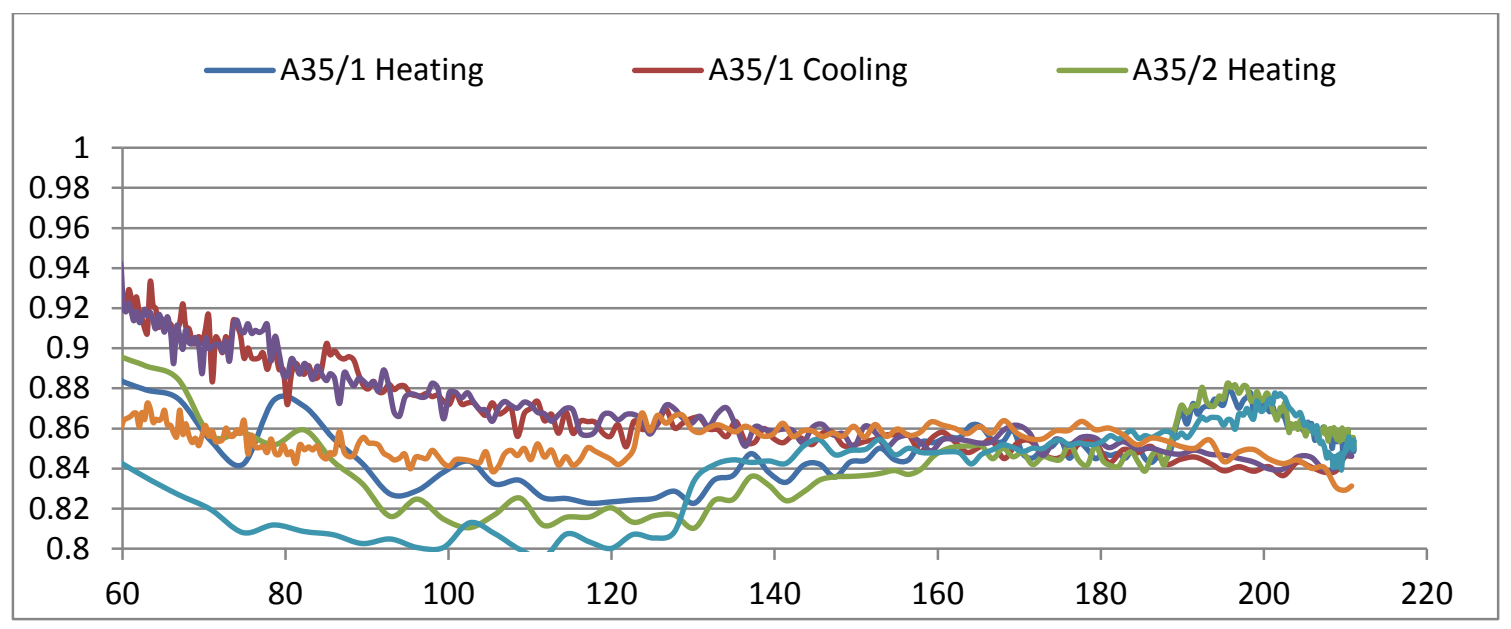

Fig. 4. Emissivity estimation in function of the temperature $\left[{ }^{\circ} \mathrm{C}\right]$ calculated from the three infrared cameras during the heating process and during the cooling.

It can be observed that there is an inconsistency of the emissivity for low temperature. However, for temperature over $100{ }^{\circ} \mathrm{C}$ the emissivity value is roughly between 0.8 and 0.85 . In the heating phase, when the CFRP reach $190^{\circ} \mathrm{C}$, a sudden rise in the emissivity can be seen. This change in emissivity correspond to the tipping temperature where the glue melt and can be either associated the latent heat or to the sudden change in the surface texture that could affect the contact between the composite sheet and the thermocouple.

Another emissivity test was performed to evaluate the difference in emissivity between a CFRP sheet that is new (never melted before) and one that has been melted beforehand. The graphs in Fig.5 present the emissivity for a 
composite sheet that has been melted before. Results showed that for the non-melted CFRP sheet, the emissivity was lower that pre-melted CFRP.

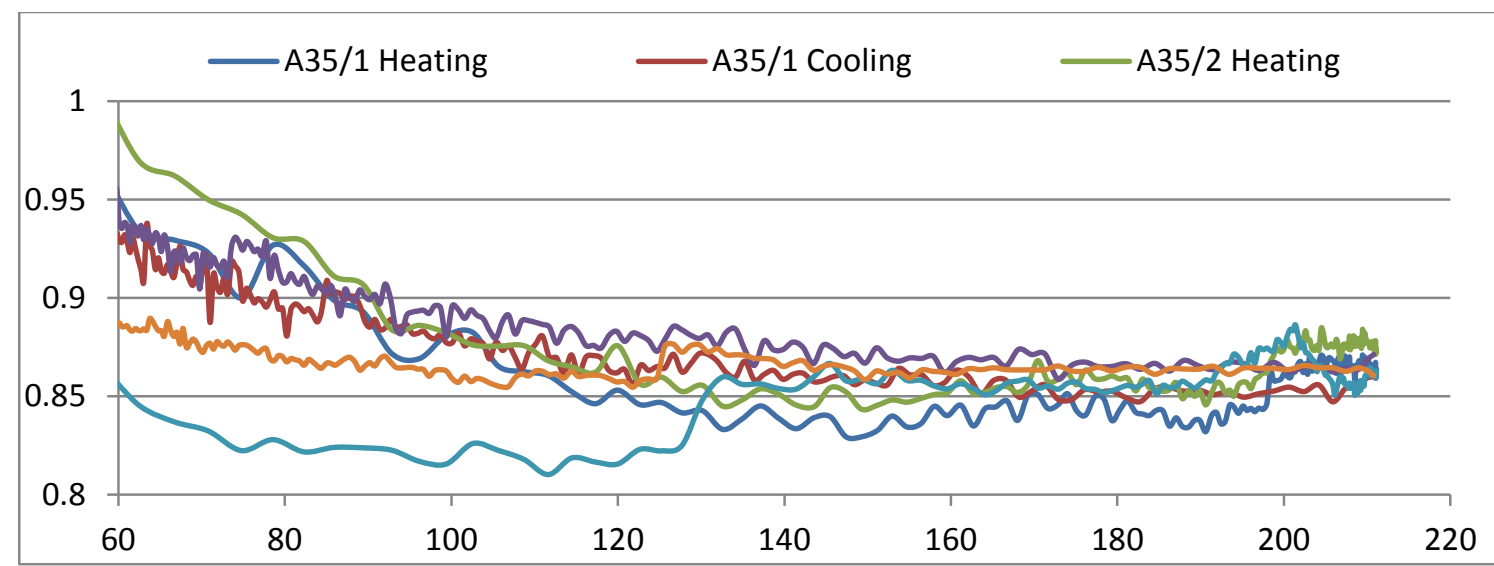

Fig. 5 Emissivity in function of the temperature $\left[{ }^{\circ} \mathrm{C}\right]$ calculated from the two infrared camera during the heating process and during the cooling for pre-melted CFRP.

Finally, as we will see in the next paragraph, the temperature interval of interest will be limited to $140^{\circ} \mathrm{C}$ to $200^{\circ} \mathrm{C}$. In that interval, the emissivity is quite stable which favour infrared measurements in a quite standard configuration.

\subsection{Phase Change}

The latent heat due to the solidification and to the melting of the thermoplastic was calculated by analyzing DSC measurements with 2 heating cycles. Results obtained are presented in table 2.

Table 2. Enthalpy variations caused by phase change measured by DSC on the thermoplastic glue.

\begin{tabular}{|c|c|c|c|c|c|}
\hline \multicolumn{3}{|c|}{ Heating } & \multicolumn{3}{|c|}{ Cooling } \\
\hline \multirow{2}{*}{\multicolumn{2}{|c|}{$\begin{array}{c}\text { Melting Temperature } \\
\text { Limits } \\
{\left[{ }^{\circ} \mathrm{C}\right]}\end{array}$}} & \multirow{3}{*}{$\begin{array}{l}\text { Normalized Latent heat } \\
\qquad\left[\mathrm{J}^{\mathrm{g}} \mathrm{g}^{-1}\right]\end{array}$} & \multirow{2}{*}{\multicolumn{2}{|c|}{$\begin{array}{l}\text { Solidification Temperature } \\
\text { Limits } \\
{\left[{ }^{\circ} \mathrm{C}\right]}\end{array}$}} & \multirow{3}{*}{$\begin{array}{l}\text { Normalized Latent heat } \\
\qquad\left[{\left.\mathrm{J} . \mathrm{g}^{-1}\right]}\right.\end{array}$} \\
\hline & & & & & \\
\hline Onset & Peak & & Onset & Peak & \\
\hline 179.2 & 191.6 & 63.9 & 165.5 & 162.2 & -39.2 \\
\hline 180.5 & 190.3 & 62.3 & 165.7 & 162.4 & -35.2 \\
\hline 179.9 & 190.9 & 63.1 & 165.6 & 162.3 & -37.2 \\
\hline 0.95 & 0.95 & 1.10 & 0.14 & 0.11 & 2.84 \\
\hline
\end{tabular}

According this table for the melting, the normalized enthalpy is $63 \mathrm{~J} / \mathrm{g}$ with a maximum enthalpy peak round $190^{\circ} \mathrm{C}$. The lower and higher limits are $170^{\circ} \mathrm{C}$ and $200^{\circ} \mathrm{C}$. For the solidification, the peak enthalpy is at $162^{\circ} \mathrm{C}$ and the limits are $143^{\circ} \mathrm{C}$ and $169^{\circ} \mathrm{C}$ with a normalized latent heat of $-37 \mathrm{~J} / \mathrm{g}$.

\section{Heating Solution Study} modeling.

This section is aimed at studying and optimizing the heating process by experimentation and numerical

\subsection{Experimental results}

To test the efficiency of the system, a dynamic test bench was put into place. The CFRP sheets are place on a conveyer that can moves at different speeds from $4.3 \mathrm{~cm} / \mathrm{s}$ to $10 \mathrm{~cm} / \mathrm{s}$ and more. The heating lamps are placed directly over the conveyer belt on which the composite sheet scrolls. Two infrared cameras (FLIR A35 series) are placed on each 
side of the lamps, however the images of only one camera (located at the outlet of the heater) are presented in the present paper. The distance between the lamp tubes and the composite was set to round $40 \mathrm{~mm}$.

Fig. 6 presents a schematic representation of the testing bench and illustrations of the CFRP sheet in place and the heating configuration.
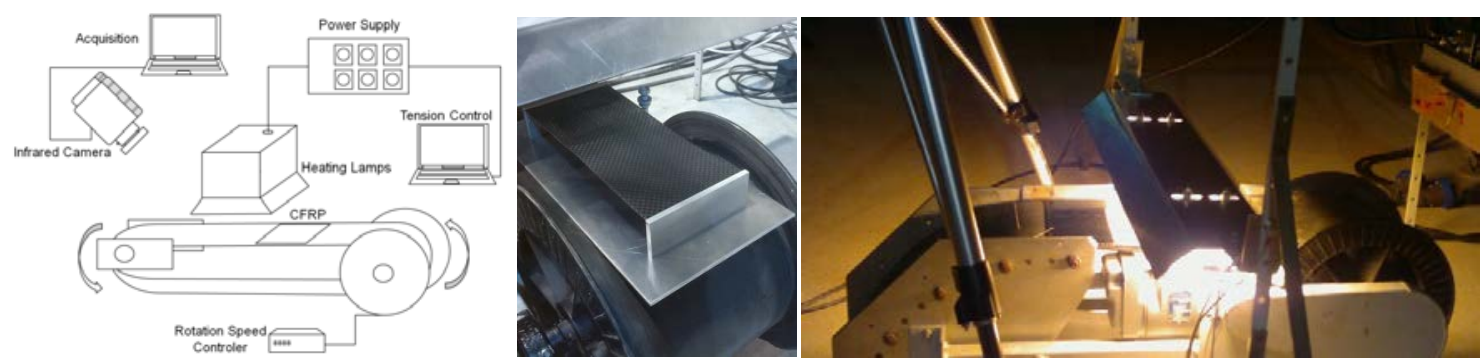

Fig. 6. From the left to the right, a diagram and pictures of the experimental setup to test the implementation of the heating process as well as the thermography system.

To improve the heating, the CFRP was placed on an aluminum support. Initial test were made where the CFRP was laying directly on the conveyer belt. This caused heat transfer between the conveyer belt and the composite sheet resulting in an overall lower temperature of the composite at the outlet of the heating. As in practice the CFRP will be welded at the outlet of the heater, this heat sink effect was solved by using a support (see picture in the middle of Fig 6).

Fig.7 shows an illustration of experimental results obtained with two Short-Wave lamps of $3 \mathrm{~kW}$. The A35 infrared camera was used for the acquisition of the infrared images.
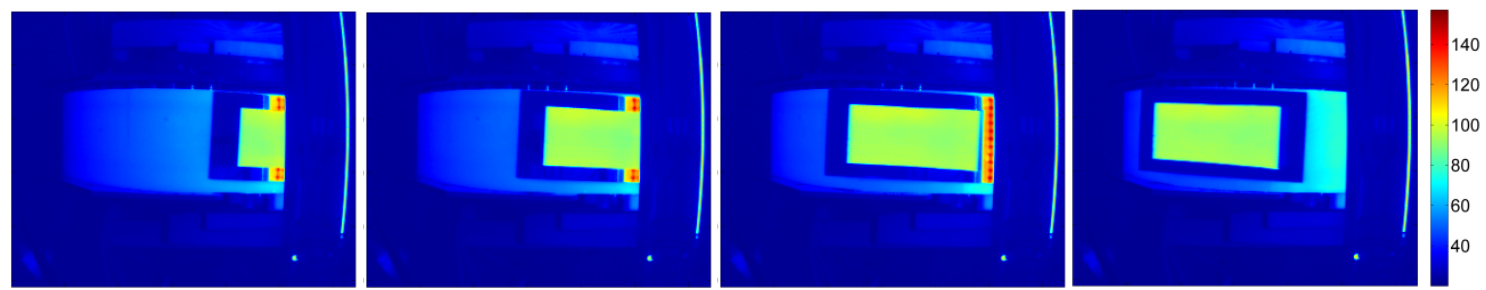

Fig. 7. Infrared thermal images taken of the CFRP sheet heated by two SW lamps. The scrolling speed is $10 \mathrm{~cm} / \mathrm{s}$

At highest speed this configuration failed to heat the composite sheet enough for the glue to melt. The average temperature reached $94^{\circ} \mathrm{C}$ (Fig. 7) and visual inspection showed no sign that any part of the sheet had melted. By reducing the speed to the minimum of $4.3 \mathrm{~cm} / \mathrm{s}$, the temperature of the CFRP reached roughly $200^{\circ} \mathrm{C}$ and caused the thermoplastic to melt. Experimental results are then used to validate and optimize the numerical modeling approach presented in next paragraph. The model will in turn allow testing different heating option and scrolling speed. The control loop of the full process will take benefit of the modeling described in paragraph 4.2.

\subsection{Numerical Model Validation}

The characterization allowed us to collect information about the CFRP properties which are used in the numerical model made with COMSOL $\odot$. The challenge in designing the model is to recreate the same heating condition and simulating the surface to surface radiation with the sample is moving. Information about the lamps is very limited. An equivalent diameter for the filaments, their emissivity and their temperature were firstly estimated and confirmed by the heating lamp manufacturer. The model takes also into account a non-constant latent heat with temperature during the melting of the thermoplastic by using eq. 2 and using values from table 2 . To validate the model in front of experimental results obtained, first simulations were undertaken for two heating lamps of $3 \mathrm{~kW}$ each. Fig. 8 present surface temperature fields calculated for a $10 \mathrm{~cm} / \mathrm{s}$ scrolling speed and different time step.
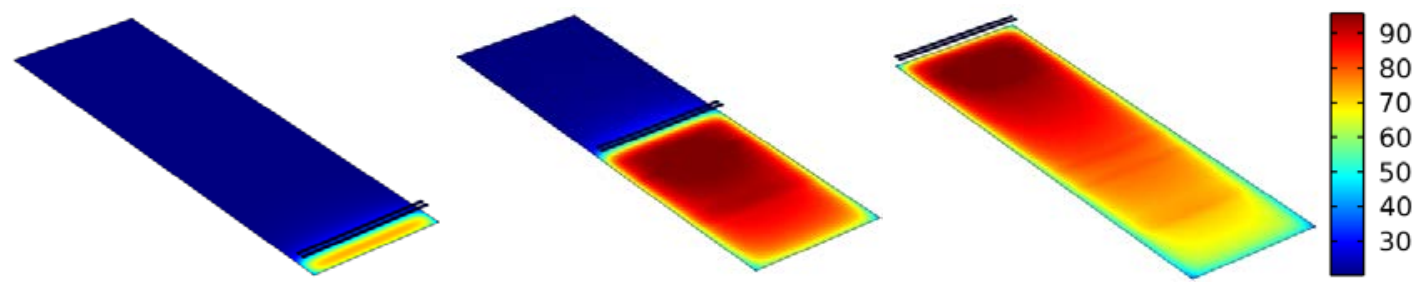

Fig 8. Simulation of the dynamic heating of two $3 \mathrm{~kW}$ SW lamps of a pre-impregnated CFRP sheet moving at 10 $\mathrm{cm} / \mathrm{s}$ at time 1,10 and 20 seconds. 
Furthermore, two distinctive state of CFRP surface studied during experiments were also considered to make the comparison with numerical simulations, one that's was already melted and a new sample. Fig. 9 presents results obtained.

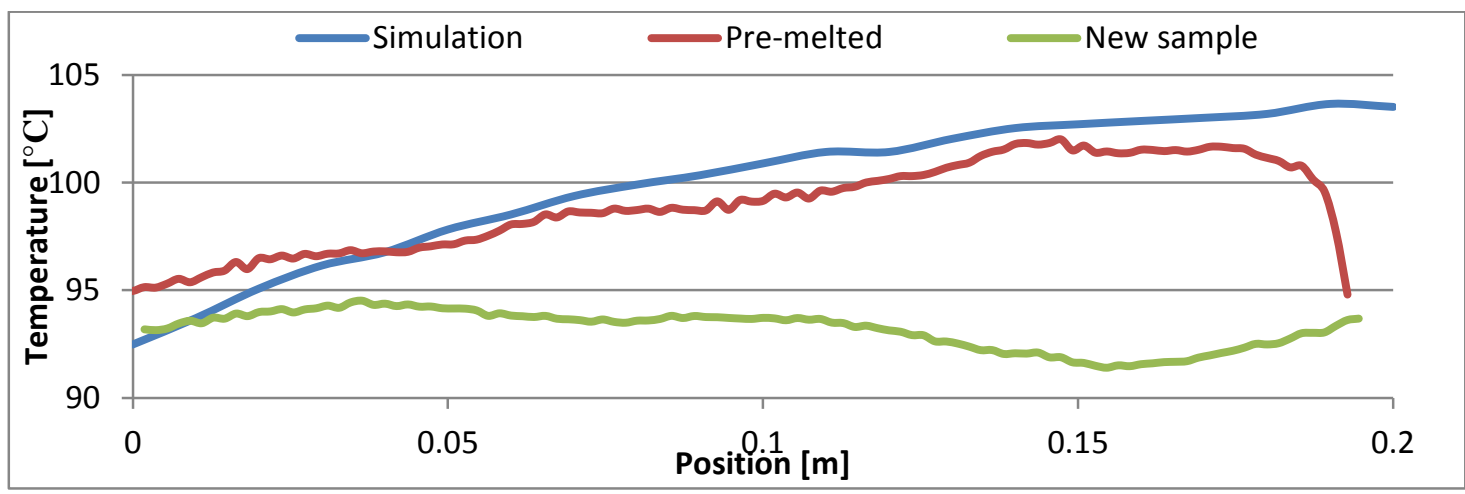

Fig. 9. Temperature profile over the length of the CFRP sheet extracted from the numerical simulation and compared to the experimental results.

Since the carbon sheet was not perfectly flat and very close to the lamps, the radiative heat flux was unevenly distributed. This is the reason why the shapes of the temperature profiles are different from the simulation. The closer the composite sheet is to the sample, the more the heat flux will be sensible to variation in the distance, as reminded in eq.4. We also notice that the sample that was already melted beforehand is significantly warmer than the new sample. This mean that the emissivity of the sheet is slightly superior once melted. This information is in accordance with the results obtained in the characterization section.

Complementary simulations were made after with a solution consisting in considering additional lamps instead of only two. The new heating lamp configuration simulated was of a higher electrical power and with active filaments of higher diameter. They are also $10 \mathrm{~cm}$ wider to tackle the problem of uneven lateral heating observed on complementary experiments on wider composite sheet. According to simulations not shown in this paper, the new set of lamps demonstrated that they could provide sufficient heating at the maximum process speed and on the whole width of the CFRP sheet.

The next step of the present study was to evaluate the effect on the temperature field evolution with time in presence of a defect. Those can be caused by the fact that the pressure applied on the CFRP is not sufficient, because the pre-impregnated glue is not heated enough to melt or if it crystallized before being installed. For now, we can assume these flaws will act as an equivalent a thin layer of air inserted in the bonding. First of all, the thermal effusivity of the CFRP sheets is only three times greater than the primer and its thickness of less than $1 \mathrm{~mm}$ means that the CFRP could quickly cool down once installed on a more cold support. So, to have a better idea of the effect of defective area thermal resistance on the temperature profile evolution, a two dimensional numerical model was conceived. It is based on a three layer geometry: CFRP, primer and concrete. A boundary condition was applied at the interface between the primer and the CFRP. The composite was at an initial temperature of $200^{\circ} \mathrm{C}$. The results from the simulation are presented in Fig.10.

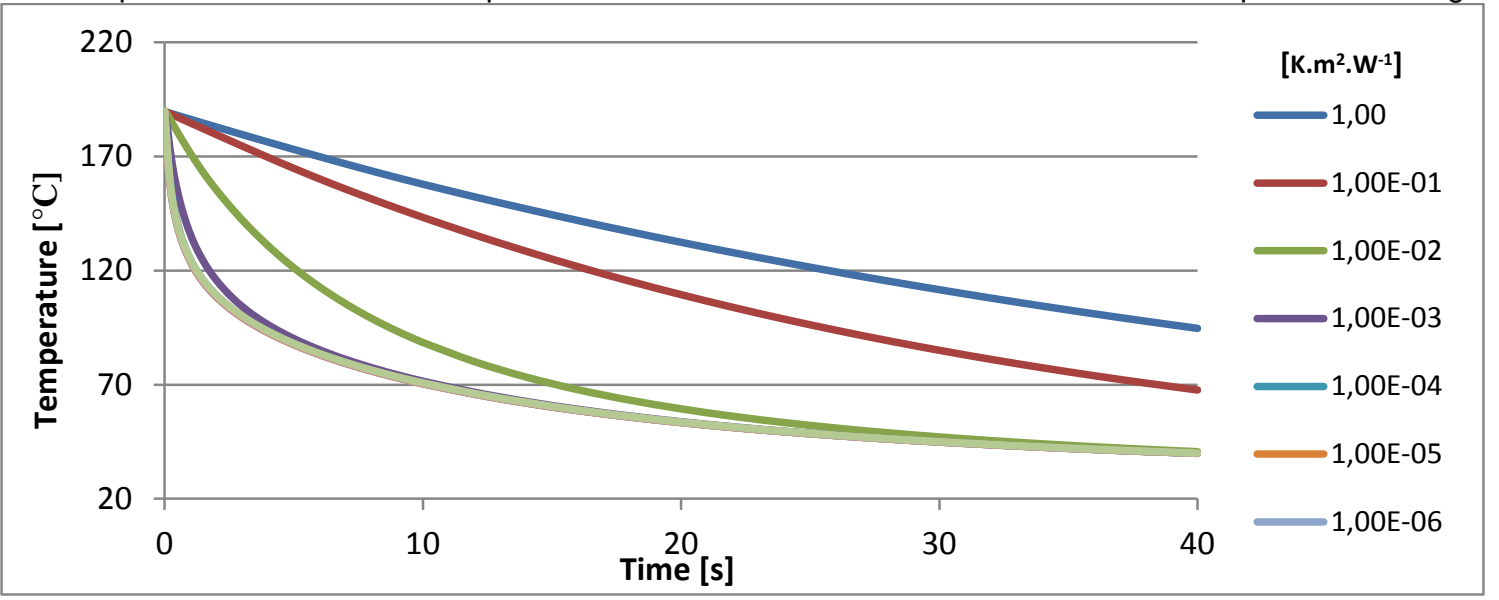

Fig. 10. Temperature profiles at the surface of the CFRP in function of the thermal resistance $\left.\left[K . \mathrm{m}^{2} . \mathrm{W}^{-1}\right)\right]$ of the simulated defects. 
By calculating the absolute difference between the reference profile (that is with no thermal resistance) and those with various thermal resistance for the defect included, it is possible to calculate the time when the contrast is maximum.

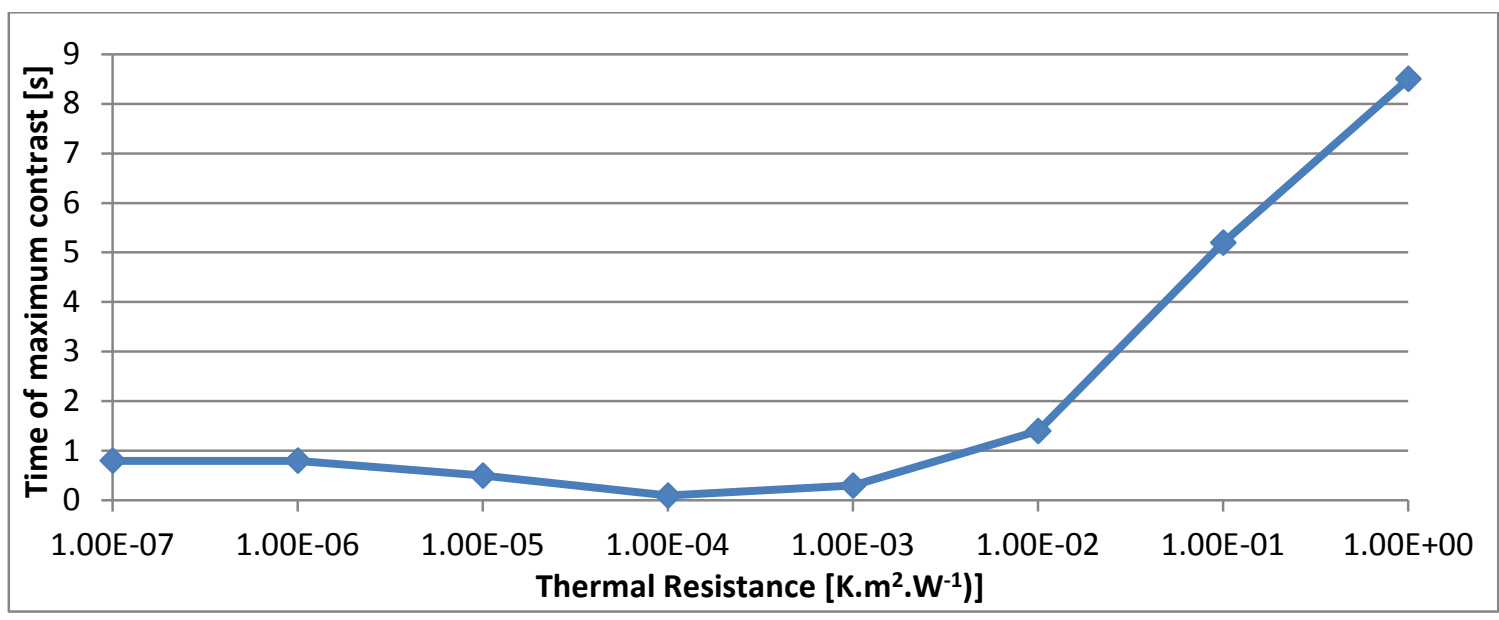

Fig. 11. Temperature profiles at the surface of the CFRP in function of the thermal resistance $\left.\left[K \cdot \mathrm{m}^{2} . \mathrm{W}^{-1}\right)\right]$ of the simulated defects.

The time of maximum contrast appearance varies greatly when the value of the thermal resistance is over 0.01 $\mathrm{K} \cdot \mathrm{m}^{2} \cdot \mathrm{W}^{-1}$. This thermal resistance correspond to a thickness of $260 \mu \mathrm{m}$ if we make the approximation that the defect can be represented by a layer of air with conductivity of $0.026 \mathrm{~W} \cdot \mathrm{m}^{-1} \cdot \mathrm{K}^{-1}$. By looking at the corresponding temperature profiles in Fig. 11, a defect with such a small thickness would still correspond to a maximum contrast of roughly $45^{\circ} \mathrm{C}$. It can be noted that the surface temperature in such process is very sensitive to the presence of thermal resistance. Since no information about the actual nature of defects expected in the real life application, the optimal placement of the infrared camera dedicated to quality gluing control still requires investigations.

\section{Conclusion}

Experiments carried out at different scrolling speeds with the SW lamps helped us to validate the numerical model. Looking at the experimental temperature profile, it can be observed that temperature reached can be different from one sample sheet of CFRP to another. This is due to the fact that the sheets were very close to the lamps, thus any deformation in the sheets caused them to be unevenly heated. Final system designed will address that problem.

The various experiments conducted in the framework of this study also showed the extent and limitation of a heating and monitoring system. The current heating system that was tested in this study showed that it was insufficient to heat the CFRP to the melting temperature at a maximum speed of $10 \mathrm{~cm} . \mathrm{s}^{-1}$. However, a numerical model of the heating system was conceived and the experimental results were be used to consolidate various parameters of the model. According to the results from the simulation, more heating tubes with a higher output power each would heat the CFRP to the desired temperature at the targeted maximum speed. Numerical simulations results showed that the reached temperature expected the requirement which leave room for unforeseen event. For optimal defect detection, a model was conceived with simulated defect. It was found that the temperature profile of the CFRP (once heated and applied on the primer) is very sensitive to the presence of thermal resistance induced by possible defect. Further experiments will be realized to confirm that trends. Anyway, for the time being, no information is available on the nature or thickness of a real defect, so the optimal placement of the quality control infrared camera still have to be optimized. In future work, it is planned to build a test bench consisting in a moving trolley that could dynamically install full width sheet over a $4 \mathrm{~m}$ long concrete stab. We are confident that experimentation with this bench will allow validation of the numerical model in regard of the predicted heating lamps by analyzing numerical simulations. It will also reveal any practical issues not anticipated and will give a good idea of the type of defects to expect for in situ application of the whole prototype.

\section{Acknowledgement}

Authors wish to thank Laurent Ibos form CERTES for his characterization of the thermal properties of the primer and prepreg CFRP.

\section{REFERENCES}

[1] ACl Committee 440.2R02-08., Guide for the Design and Construction of Externally Bonded Systems for Strengthening Concrete Structures, American Concrete Institute, Michigan, U.S.A., 2008. 
[2] Fib Task Group 9.3. Externally bonded FRP reinforcement for $R C$ structures, fib bulletin 14, Lausanne, Switzerland, 2001.

[3] Balageas D.L., Deom A.A., Boscher D.M., Characterization and nondestructive testing of carbon-epoxy composites by a pulsed photothermal method, Journal of Materials Evaluation, vol. 45, $\mathrm{n}^{\circ}$ 4, 1987, p. 465-466.

[4] X.P.V. Maldague, Theory and practice of infrared technology for non-destructive testing, John Wiley \& sons Inc., 2001.

[5] Dumoulin J., Taillade F., Benzarti K., Quiertant M., Aubagnac C., Infrared Thermography for the Nondestructive Inspection of CRFP Strengthening, Concrete International, April 2011, 54-58

[6] Crinière A., Dumoulin J., Ibarra-Castanedo C. and Maldague X., Inverse model for defect characterization of externally glued CFRP on reinforced concrete structures: Comparative study of square pulsed and pulsed thermography, Quantitative InfraRed Thermography Journal, Taylor \& Francis Editor, 2014. DOI: 10.1080/17686733.2014.897512.

[7] Siegel R. and Howell J., Thermal Radiation Heat Transfer, Fourth Edition, Taylor and Francis editor, 2002.

[8] Hladik J., Métrologie des propriétés thermophysiques des matériaux, Masson editor, 1990.

[9] Monchau J-P., Mesure d'émissivité pour la thermographie infrarouge appliquée au diagnostic quantitatif des structures, PhD Thesis, Paris-Est University, 2013

[10] Boudenne A., Ibos L., Géhin E., Candau Y., "A simultaneous characterisation of thermal conductivity and diffusivity of polymer materials by a periodic method", Journal of Physics D : Applied Physics, vol. 37(1), 2004, p. 132-139.

[11] Kelsall D., An automatic emissivity-compensated radiation pyrometer, Journal Scientific Instruments, IOP editor, Vol 40, 1963.

[12] Dumoulin J., Mesure du coefficient d'échange pariétal dans le cas d'un refroidissement par impact de jet, Master Thesis, INSA de Toulouse, 1991. 